\title{
Excitation Spectra and Thermodynamic Response of Segmented Heisenberg Spin Chains
}

\author{
Stefan Wessel and Stephan Haas \\ Department of Physics and Astronomy, University of Southern California, Los Angeles, CA 90089-0484
}

(October 25, 2018)

\begin{abstract}
The spectral and thermodynamic response of segmented quantum spin chains is analyzed using a combination of numerical techniques and finite-size scaling arguments. Various distributions of segment lengths are considered, including the two extreme cases of quenched and annealed averages. As the impurity concentration is increased, it is found that (i) the integrated spectral weight is rapidly reduced, (ii) a pseudo-gap feature opens up at small frequencies, and (iii) at larger frequencies a discrete peak structure emerges, dominated by the contributions of the smallest cluster segments. The corresponding low-temperature thermodynamic response has a divergent contribution due to the odd-site clusters and a sub-dominant exponentially activated component due to the even-site segments whose finite-size gap is responsible for the spectral weight suppression at small frequencies. Based on simple scaling arguments, approximate low-temperature expressions are derived for the uniform susceptibility and the heat capacity. These are shown to be in good agreement with numerical solutions of the Bethe ansatz equations for ensembles of open-end chains.
\end{abstract}

\section{INTRODUCTION}

Low-dimensional electron systems are known to be particularly sensitive to disorderl. It is therefore difficult to realize pure one- or two-dimensional behavior at very low temperatures and small frequencies in nature. 2 This is somewhat disappointing in light of recent detailed theoretical predictions for the low-energin scaling behavior of paradigmatic quantum-many-body models, such as antiferromagnetic spin chains and ladder $\mathbf{B}$. It is the enhanced quantum fluctuations in these compounds, which give rise to particular low-temperature scaling regimes in very pure samples, and at the same instance make them highly unstable towards externally induced low-temperature transitions, such as localization by impurity scattering or three-dimensional long-range ordering due to residual small couplings between the lower-dimensional subsystems

It has recently been demonstrated that in certain $1 \mathrm{D}$ subsystems, such as random-exchange and random-spin chains, anomalously extended states can persist against disorder 10 14. The physical picture is that while most spins are bound in randomly distributed valence bgnds, the unbound spins interact via virtual excitations, resulting in a zero-frequency band with power-law scaling. 15 Furthermore, in the case of spin ladders and spin-Peierls compounds, doping with randomly placed non-magnetic impurities may actually indmce quasi-long-range ordering due to effective inverse-power-law interactions between the residual "pruned" spins 16.47 . This replacement of an originally shortranged spin liquid state by impurity-induced quasi-long-range order can be viewed as a realization of the "order by disorder" phenomenon.

In other compounds, impurity scattering may completely destroy the connectivity within one-dimensional subsystems. If this is the case extended states cannot survive. Let us examine two specific realizations of such segmented quantum spin systems: (i) $\mathrm{CuO}$ chains, with non-magnetic impurities, and (ii) pinned charge density waves in quasi$1 \mathrm{D}$ materials. The first situation can be realized by doping a quasi-1D compound such as $\mathrm{SrCuO}_{2}$ with $\mathrm{Zn}$. In the pure material, antiferromagnetic superexchange between neighboring $\mathrm{Cu}^{2+} \mathrm{d}_{\mathrm{x}^{2}-\mathrm{y}^{2}}$ electrons is mediated by the filled $\mathrm{O}^{2-}$ p-orbitals. By substituting $\mathrm{Zn}^{2+}$ for $\mathrm{Cu}^{2+}$, static wacancies are created, and the infinite chain is separated into segments of length $l$ which follow a discrete distribution.18.19 A second physical way of realizing segmented spin chains is the pinning of one-dimensional charge density waves. If there is competition between poorly screened long-ranged repulsive Coulomb forces and short-range attractive forces, highly inhomogeneous density wave modulations occur, favoring particular segment lengths. 20 Even small impurity scattering leads to a pinning of such structures.

In both cases, there are ensembles of correlated spin segments which are most straightforwardly modelled by taking appropriate averages over distributions of finite clusters with open boundary conditions. The specific form of the distribution function strongly depends on the details of how the segments are formed. For example, in the case of randomly doped $\mathrm{CuO}$ chains a (discrete) Poisson distribution is natural 18.19 , whereas for pinned, spatially modulated density waves, only two or three cluster sizes may dominate. An important factor, determining the proper distribution function, is the (meta-)stability of the random realizations: are they obtained from a quenched or an annealed cooling 
procedure? While in quenched realizations all clusters that occur at high temperatures also have non-vanishing weight in the zero-temperature distribution function, slower "annealed" cooling processes can lead to preferred sizes and shapes. In particular, segments with an electronic closed shell configuration have more stable groundstates than others, and thus receive a higher weight in an annealed cooling process 21

In this work, we systematically study such ensembles of antiferromagnetically correlated spin clusters, focusing on the evolution of the corresponding low-energy features in the dynamical spin excitation spectrum and on the uniform static susceptibility as a function of the hole concentration. In particular for small clusters, quantum effects are important21, which makes any theoretical approach to this problem challenging. We therefore attack this task numerically, using exact numerical diagonalization 22 and scaling laws derived from conformal field theory to calculate the static and dynamical magnetic response for variable segment sizes 18 .

This paper is organized as follows. In the next section, a procedure to obtain excitation spectra for segmented Heisenberg chains is explained. The appropriate distribution functions are derived, and the evolution of the spectra with impurity concentration is discussed. In the subsequent section, the static magnetic response, i.e. the uniform susceptibility, and the heat capacity are calculated for various ensembles of finite chains. In the final section we conclude with a discussion of possible extensions and experimental consequences of the procedure outlined in this paper.

\section{EXCITATION SPECTRA AT $\mathrm{T}=0$}

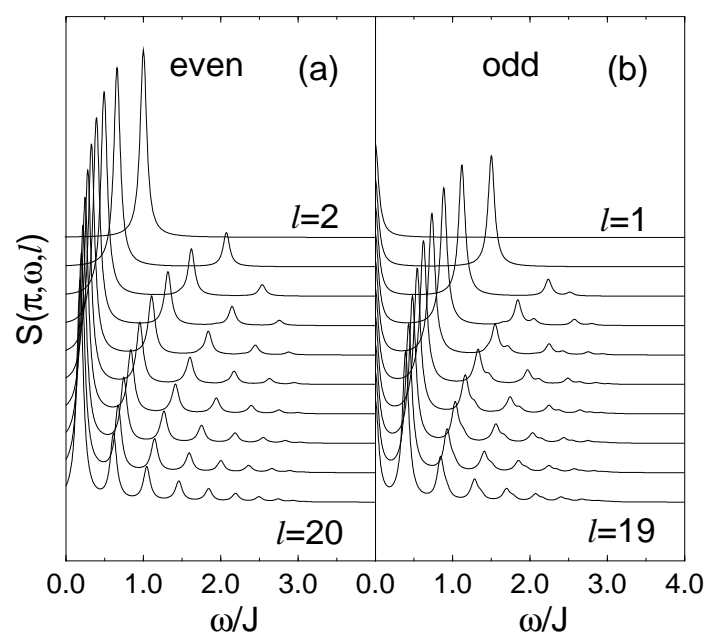

FIG. 1. Finite-size scaling of the staggered dynamical structure factor of the antiferromagnetic spin- $\frac{1}{2}$ Heisenberg chain with open boundary conditions. The poles have been given a width of $\epsilon=0.1 J$. (a) chains with an even number of sites, (b) chains with an odd number of sites.

To study the zero-temperature dynamical response of randomly segmented Heisenberg chains we examine the dynamical structure factor $S(q, \omega, l)$ of finite chains with open boundary conditions:

$$
S(\mathbf{q}, \omega, l)=\frac{1}{l Z} \sum_{n=1}^{l}|<n| S_{\mathbf{q}}^{z}|0>|^{2} \delta\left(\omega-E_{n}+E_{0}\right),
$$

where $l$ is the length of the finite chain, $Z$ the partition function and $n$ runs over all possible final states, whereas $\mid 0>$ is the groundstate. Let us first concentrate on the staggered magnetization

$$
S_{\pi}^{z}=\sum_{n=1}^{l}(-1)^{n} S_{n}^{z}
$$

Then $S(\pi, \omega, l)$ is well defined for open boundary conditions and chains of an even or odd number of sites $l$. In the case of even $l$ there is a unique singlet groundstate, whereas one has to take into account the doublet nature of the groundstate for odd $l$. Using numerical diagonalization techniques, we have obtained $S(\pi, \omega, l)$ with $l=1, \ldots, 20$. The resulting spectra are shown in Fig. 1. We observe that segments of even length $l$ have $l / 2$ major peaks at non-zero 
frequencies, whereas those of odd length have a significant pole at $\omega=0$ and $(l-1) / 2$ additional peaks at higher frequencies. The higher energy peaks have a more complex structure, because some of the final states are singlets. Zero-frequency peaks occur only in the odd length segments, reflecting the fact that their groundstates are doublets.

As in the case of closed finite chain 18 equations derived from conformal field theory can be used to extract the finite-size scaling behavior of $S(\pi, \omega, l) .2326$ To leading order, the pole positions and amplitudes are given by 18,26

$$
\begin{aligned}
& \omega_{i}(l)=\alpha_{i} / l+\beta_{i} / l[\ln (l)], \\
& A_{i}(l)=a_{i}+b_{i} \ln (1+l)+c_{i} \ln [1+\ln (1+l)],
\end{aligned}
$$

where the coefficients $\alpha_{i}, \beta_{i}, a_{i}, b_{i}$, and $c_{i}$ can be treated as fit parameters. In Fig. 2 the pole positions and amplitudes of the lowest three poles are shown for clusters of up to 20 sites, along with the fits to the above scaling equations. In particular for the larger-size segments, these equations give an excellent fit to the numerical data. In the shorter segments higher-order logarithmic corrections for the amplitudes become increasingly relevant, and the quality of the fits deteriorates slightly in this regime. Note also, that the peak of the one-site cluster at $\omega=0$ has an amplitude that does not follow the general trend. The amplitudes shown in Fig. 2 correspond to the dynamical spin response per site (Eq. (1)). In the following, we will consider averages over ensembles of finite chains, where the amplitudes of the individual segments enter as extensive quantities. In this case, the segment amplitudes per site shown in Fig. 2 have to be multiplied by the segment length 1 (Eqs. 5 and 6 ).

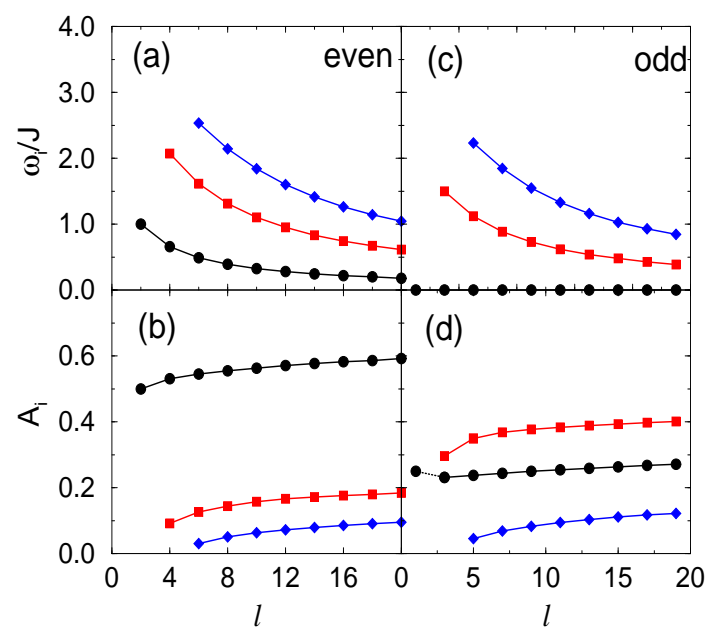

FIG. 2. Finite-size scaling of the first three poles of the staggered dynamical structure factor of spin- $\frac{1}{2}$ Heisenberg chains with open boundary conditions. The symbols show the values obtained by numerical diagonalizations, and the solid lines are the result of a fit to equations (3) and (4). Circles: first poles, squares: second poles, diamonds: third poles. (a) pole positions for chains of even length, (b) the corresponding amplitudes, (b) and (d) analogous results for chains of odd length.

Now consider an infinite Heisenberg chain, doped with non-magnetic impurities, resulting in an ensemble of open-end segments of various lenghts 1 . The average dynamical spin structure factor can then be calculated from

$$
S(\pi, \omega)=\sum_{l} l P(l) S(\pi, \omega, l),
$$

where $P(l)$ is an appropriately chosen distribution function. Using the pole structure of the response functions for the individual segments, we obtain

$$
S(\pi, \omega)=\sum_{l} \sum_{i} l P(l) A_{i}(l) \delta\left(\omega-\omega_{i}(l)\right) .
$$

In practice, the $\delta$-function in Eq. 6 is replaced by a Lorentzian of width $\epsilon$, which will be taken as $\epsilon=0.1 J$ throughout the paper. $P(l)$ determines the weight of each segment in the ensemble average, and extrinsic factors favoring certain cluster shapes over others enter through this function. If the chain segmentation occurs completely randomly, the corresponding distribution function is given by $P(l)=\rho^{2}(1-\rho)^{l}$, where $\rho \in[0,1]$ is the concentration of vacant sites 19 27. This distribution function is normalized by

$$
\sum_{l=1}^{\infty} l P(l)=1-\rho
$$


and for $\rho \ll 1$ it can be approximated by $P(l) \approx \rho^{2} \exp (-\rho l)$. We also note that since the total number of clusters per site is given by $n_{c}=\sum_{l} P(l)=\rho(1-\rho)$, the average length of the clusters is $l_{a v}=\sum_{l} l P(l) / n_{c}=1 / \rho$. The above distribution describes the case of quenched disorder in the infinite chain, i.e. the positions of the impurities are uncorrelated. In an annealed cooling process, even length segments are favoured over odd ones, because they have a lower groundstate energy. We describe this situation by a similar distribution function $P_{a}(l)=C \rho^{2}(1-\rho)^{l} \delta_{0, l \bmod 2}$ where $\mathrm{C}$ is determined by the normalization condition (7). Only even length spin segments occur in a chain with this distribution function for the impurities. Note, that according to common terminology all of the ensemble averages which are discussed here are "quenched" because frozen disorder realizations are used. In this paper, the terms "annealed" and "quenched" only refer to the cooling procedures giving rise to different distribution functions. For true annealed disorder, however, the disorder variables would have to be treated dynamically.

Here we wish to evaluate and analyze $S(\pi, \omega)$ and $S(\pi)$ by using the finite-size scaling behavior of the lowest few poles in the spectrum. This few-mode approximation has been shown to be valid for sufficiently large impurity concentrations, but it tends to underestimate the spectral response for very small $\rho .18$ Let us use the lowest three poles of each segment as shown in Fig. 2, i.e. the index $i$ in Eq. (6) runs from $i=1$ to 3. Also, a cutoff length $l_{\max }=10000$ is used in the sum over $l$. The resulting frequency-integrated staggered dynamical structure factor $S(\pi)=\int d \omega S(\pi, \omega)$ is then given by

$$
S(\pi)=\sum_{l=1}^{l_{\max }} l P(l) S(\pi, l),
$$

where $S(\pi, l)$ is the frequency-integrated dynamical structure factor at wavevector $\pi$, with the scaling form

$$
S(\pi, l)=a+b \ln (1+l)+c \ln [1+\ln (1+l)] .
$$

Comparing the values of $S(\pi)$ obtained from exact numerical diagonalizations of ensembles of finite clusters with the result for $S(\pi)$ within the three-mode approximation, one finds that the three-mode approximation tends to underestimate $S(\pi)$ for small impurity concentrations. The difference in the integrated weight is due to neglecting the higher frequency poles that become more relevant for larger clusters and thus smaller impurity concentrations. This weight can be approximately restored by adding the properly normalized dynamical structure factor of an infinite Heisenberg chain, broadend by $\epsilon$ to $\Delta S(\pi, \omega) \propto \epsilon /\left(\omega^{2}+\epsilon^{2}\right) / \pi$, neglecting logarithmic corrections. It turns out that these corrections to $S(\pi)$ are only relevant for very low impurity concentrations and are neglegible for $\rho>0.2$. The resulting spectra are shown for different $\rho$ in Fig. 3 for the quenched and the annealed case.

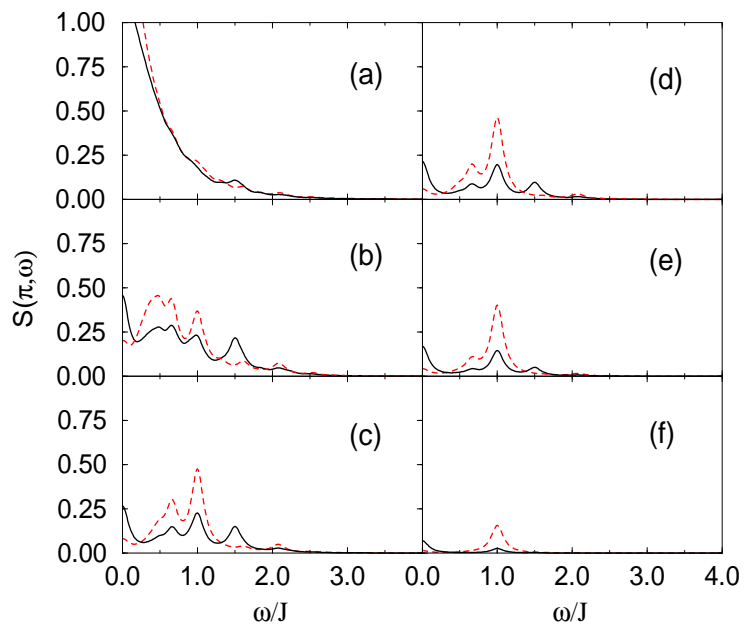

FIG. 3. Evolution of $S(\pi, \omega)$ of the segmented antiferromagnetic spin- $\frac{1}{2}$ Heisenberg chain as a function of the impurity concentration. (a)-(f) spectra for $\rho=0.1,0.3,0.5,0.6,0.7,0.9$. The solid lines represent the quenched case, and the dashed lines represent the annealed case.

For both types of impurity distribution functions there are two main features that occur in $S(\pi, \omega)$ upon increasing the number of vacancies in the infinite chain. First, the integrated spectral weight decreases rapidly upon increasing the impurity concentration. This is shown explicitly in Fig. 4, where the solid lines in part (a) and (b) represent the dependence of $S(\pi)$ on $\rho$. Note that in the annealed case the integrated spectral weight is decreasing at a slower rate than in the quenched case, especially for large impurity concentrations. This is due to the fact, that the integrated 
spectral weight of finite chains is a monotonous function of $l$. Thus the total response increases if at constant impurity concentration the odd-length segments (starting with $l=1$ ) are substituted by even-length segments (starting with $l=2$ ). Most of the suppressed spectral weight comes from the low-energy continuum of the response function due to the large segments. It can be shown by considering a lowest-order single-mode approximation that $S(\pi, \omega)$ is in fact exponentially suppressed at low frequencies 18 .

The second common feature is the emergence of a discrete peak structure at larger frequencies (of order J), dominated by the contributions of the smallest cluster segments. The dominant segments, occuring according to the distribution function $P(l)$, can be identified from these higher energy peaks. In the annealed case, the smallest segments are the two-site clusters with a pole in $S(\pi, \omega, l)$ at $\omega=1 J$, and the four-site chain with a major pole at $\omega=0.66 J$. These poles are well separated from the low-energy continuum and carry most of the spectral weight of $S(\pi, \omega)$ at impurity concentrations $\rho \geq 0.5$ (Fig. 3 (c) - (e)). The pole of the smallest segment at $\omega=1 J$ dominates the dynamical response function in the annealed case at high concentrations, $\rho>0.7$. One thus expects that most of the spectral weight can be obtained from the two-site cluster for $\rho$ close to one. In fact, from Eq.(8) one finds

$$
S(\pi) \approx 2 P(2) S(\pi, 2) \approx \frac{1-\rho}{2}, \quad \rho \rightarrow 1,
$$

explaining the linear behavior of $S(\pi)$ for large impurity concentrations. Since in the quenched case the single spin sites and the three-site chains are also present, their low frequency poles contribute strongly to the spectral weight. The major pole of the three site chain at $\omega=1.5 \mathrm{~J}$ is the first well-defined pole to separate from the low-energy continuum upon increasing the impurity concentration, as can be seen in Fig. 3 (a) already at $\rho=0.1$. It dominates the spectrum away from the low-energy pseudo-branch until, at intermediate concentrations, the lowest-energy poles of the two- and four-site clusters are also separated from the continuum. Characteristic features of the underlying impurity distribution can thus be read off from the higher frequency part of the spectrum.

The major difference between the two impurity distributions we have studied lies in the rate at which $S(\pi, \omega)$ is suppressed for small frequencies. Consider the annealed case first. The even-site segments do not have a pole at $\omega=0$ due to their inherent finite spin-gap. Thus the exponential suppression of the contributions from the large segments leads to the development of a pseudogap at small frequencies with increasing impurity concentration. The finite values of $S(\pi, 0)$ are due to our replacement of the $\delta$-peak in Eq. (6) by Lorentzians. This mimics the various broadening mechanisms in real materials, such as thermal broadening, scattering from phonons, and interactions with out-of-chain impurities. As shown in Fig. 4 (c), the residual spectral weight $S(\pi, 0)$ is exponentially suppressed with increasing $\rho$. In Fig. 4 (d) the important higher-energy peaks are compared to $S(\pi, 0)$, clearly indicating the reduction of the zero-frequency weight in the annealed case.

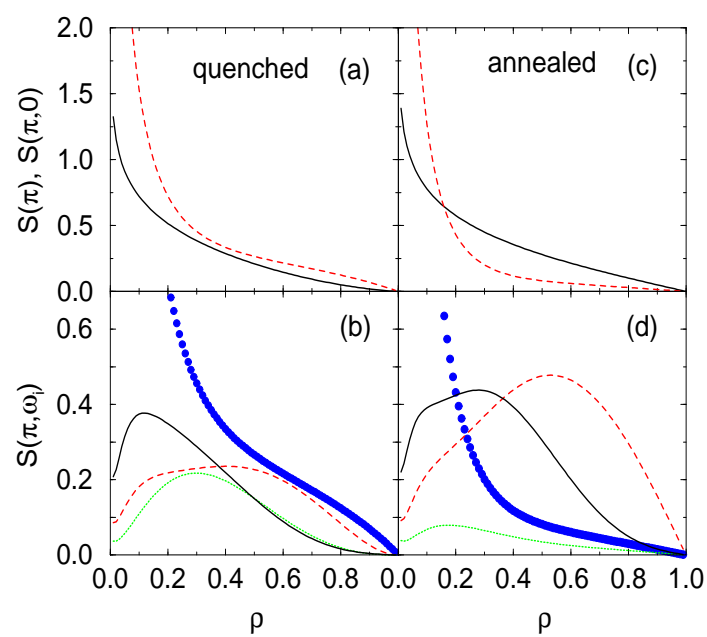

FIG. 4. (a) and (c) The frequency-integrated dynamical structure factor at wavevector $\pi, S(\pi)$ (solid line) and the residual spinon density of states $S(\pi, 0)$ (dashed line) as a function of the impurity concentration $\rho$. (a) quenched disorder, (c) annealed disorder. (b), (d) Dependence of the amplitudes of the peaks at $\omega=0$ (full circles) $\omega_{1}=0.66 J$ (solid line), $\omega_{2}=1.0 J$ (dashed line) and $\omega_{3}=1.5 \mathrm{~J}$ (dotted line) on the impurity concentration $\rho$. (c) quenched, (d) annealed.

Now consider the quenched case. All odd-length segments and especially the dominating one-spin segment show a pole in their dynamical response function at zero frequency. Therefore one expects the rate of suppression of $S(\pi, 0)$ to be significantly reduced in the quenched case with respect to the annealed case. This is clearly observed in Fig. 4 , comparing the value of $S(\pi, 0)$ for both distributions. In the quenched case the weight at $\omega=0$ remains a major 
contribution to $S(\pi)$, even at large impurity concentrations. Fig. 4 (a) shows that the reduction of $S(\pi, 0)$ with increasing $\rho$ is less pronounced in the quenched case. In fact, the amplitude of the peak at $\omega=0$ dominates the spectrum for all $\rho$, as shown in Fig 4. (b).

\section{THERMODYNAMIC RESPONSE: UNIFORM SUSCEPTIBILITY AND HEAT CAPACITY}

In this section we examine the thermodynamic response of segmented spin- $1 / 2$ Heisenberg chains. Based on simple scaling arguments, low-temperature approximations for the uniform susceptibility and the specific heat are derived and shown to be in good agreement with a numerical solution of the problem, using the Bethe ansatz equations for open-end chains. The impurity tail of the low-temperature susceptibility (i.e. the divergence of $\chi(T)$ at $T=0)$ in an ensemble of segmented chains is caused by the odd-length segments. Before examining the sub-dominant contributions of the even-length segments it is therefore necessary to first identify and discuss the dominant divergent contributions of the odd-length clusters. This can be achieved by analyzing $[\chi(T) T]$. Its value at zero temperature, $\lim _{T \rightarrow 0}[\chi(T) T]$, gives the prefactor of the low-temperature impurity tail in $\chi(T)$. For chains of even length, which always have a finite spin gap, the value of $[\chi(T) T]$ approaches 0 as $\mathrm{T}$ goes to zero, whereas for odd lengths $l, \lim _{T \rightarrow 0}[\chi(T) T]=1 /(4 l)$. It follows that in the quenched case

$$
[\chi(T) T]_{0}(\rho) \equiv \lim _{T \rightarrow 0}[\chi(T) T](\rho)=\lim _{T \rightarrow 0} \sum_{l=1}^{\infty} l P(l)[\chi(T) T](l)=\frac{\rho(1-\rho)}{4(2-\rho)},
$$

with a maximum at $\rho=2-\sqrt{2} \approx 0.586$. In the ideal annealed case only even-length segments contribute to the average, and therefore $[\chi(T) T]_{0}(\rho)$ vanishes. The Curie constant is $C(\rho)=\lim _{T \rightarrow \infty}[\chi(T) T]=(1-\rho) / 4$, independent of the length of the contributing segments.

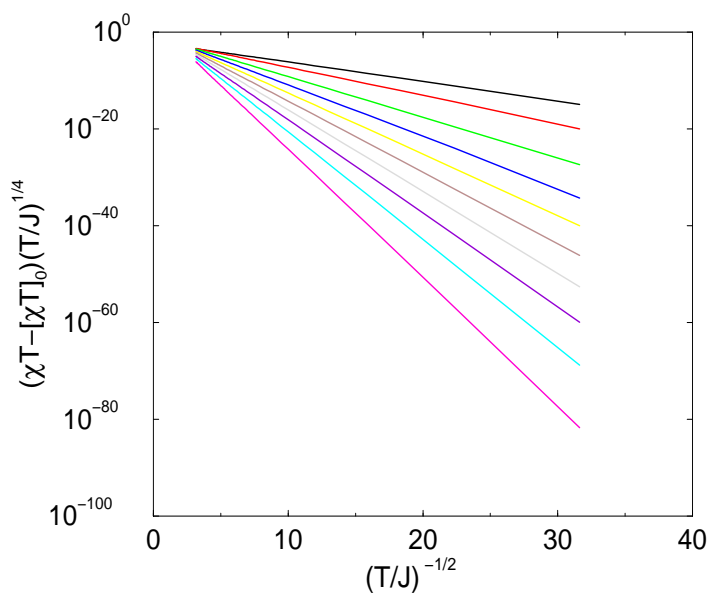

FIG. 5. Linearized form of the scaling behaviour for the low temperature susceptibility of segmented Heisenberg chains for impurity concentrations $\rho=0.05$ (top) $, 0.1,0.2, \ldots, 0.9$ (bottom).

To study the temperature dependence of $\chi(T)$ we can calculate the finite-size susceptibilities from the Bethe ansatz equations which determine the energy levels of open Heisenberg chains:

$$
\left(\frac{x_{k}+i}{x_{k}-i}\right)=\prod_{j \neq k}^{M} \frac{x_{k}-x_{j}+2 i}{x_{k}-x_{j}-2 i} \frac{x_{k}+x_{j}+2 i}{x_{k}+x_{j}-2 i}
$$

Here the number of roots $\mathrm{M}$ determines the total $S^{z}$ component of the state through the relation $S^{z}=l / 2-M$. In logarithmic form this equation becomes

$$
2 l \operatorname{ctan}^{-1}\left(x_{k}\right)=\pi I_{k}+\sum_{j \neq k}^{M}\left[\operatorname{ctan}^{-1}\left(\frac{x_{k}-x_{j}}{2}\right)+\operatorname{ctan}^{-1}\left(\frac{x_{k}+x_{j}}{2}\right)\right]
$$


where all the $I_{k}$ are integers with $k=1, \ldots, M$. Given a solution of the above equations for a set of $I_{k}$ 's, the energy of the corresponding eigenstate is

$$
\frac{E}{J}=\frac{l-1}{4}-2 \sum_{k=1}^{M} \frac{1}{x_{k}^{2}+1}
$$

The groundstate in a given $S^{z}$ sector, $E_{0}\left(S^{z}, l\right)$, is obtained from the set $\left\{I_{k}\right\}=\{l+1, l+3, l+5, \ldots, l+2 M-1\}$. And the first exited state in the $S^{z}>0$ sectors can be determined from the set $\left\{I_{k}\right\}=\left\{2 S^{z}, 2 S^{z}+3,2 S^{z}+5,2 S^{z}+7, \ldots, l-1\right\}$. Using an iterative method to determine the energy gaps for the even length segments, $\Delta E_{l}=E_{0}\left(S^{z}=1, l\right)-E_{0}\left(S^{z}=\right.$ $0, l)$, one obtains for the low temperature susceptibility of the segmented chain:

$$
[\chi(T) T](\rho)=[\chi(T) T]_{0}(\rho)+2 \sum_{l=2}^{\infty}, \frac{P(l)}{1+\exp \left(\Delta E_{l} / T\right)},
$$

where the sum is restricted to even $l$. This equation can be evaluated numerically, using the energies from the finitesize Bethe ansatz equations (Eq. 14) and an appropriate distribution function $P(l)$. It is found that the results for $[\chi(T) T]$ typically converge below a length cut-off around $l_{\max }=1000$. Results for the quenched case are shown in Fig. 5. For low temperatures there appears to be a universal scaling behavior which can be elucidated by expanding Eq. 15.

To determine the low-temperature scaling behavior of the susceptibility we consider the lowest-order finite-size scaling behavior of the energy gap, $\Delta E_{l} \approx \alpha_{1} / l$, neglecting higher-order logarithmic corrections (Eq. 3) which become increasingly important when larger segments occur (i.e. at very small impurity concentrations). For low temperatures one obtains

$$
\begin{aligned}
{[\chi(T) T](\rho)-[\chi(T) T]_{0}(\rho) } & \approx \int_{0}^{\infty} \exp \left[-\frac{\alpha_{1}}{T l}-\ln \left(\frac{1}{1-\rho}\right) l\right] d l=\sqrt{\frac{4 \alpha_{1}}{T \ln \left(\frac{1}{1-\rho}\right)}} K_{1}\left[\sqrt{\frac{4 \alpha_{1}}{T} \ln \left(\frac{1}{1-\rho}\right)}\right] \\
& \approx \sqrt{\frac{\pi}{2} \sqrt{\frac{\alpha_{1}}{T}}}\left[\ln \frac{1}{1-\rho}\right]^{-\frac{3}{4}} \exp \left[-\sqrt{\frac{4 \alpha_{1}}{T} \ln \left(\frac{1}{1-\rho}\right)}\right], \quad T \rightarrow 0 .
\end{aligned}
$$

The expected low-temperature behavior is thus of the form

$$
[\chi(T) T](\rho)-[\chi(T) T]_{0}(\rho) \propto\left(\frac{T}{J}\right)^{-\frac{1}{4}} e^{-\gamma / \sqrt{T / J}},
$$

where $\gamma$ turns out to be

$$
\gamma=\sqrt{4 \frac{\alpha_{1}}{J} \ln \left(\frac{1}{1-\rho}\right)} .
$$

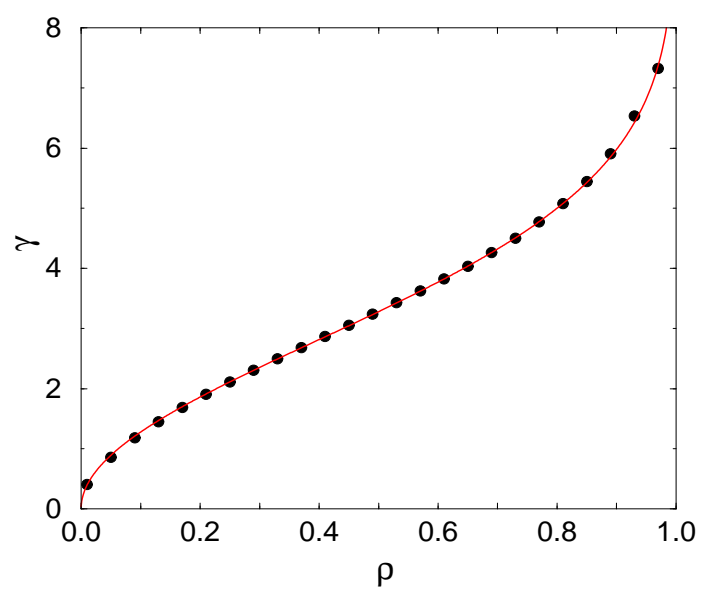

FIG. 6. Fit of the effective low-temperature gap $\gamma$. The circles show the numerical solution of the Bethe ansatz equations, and the solid line is the best fit with $\alpha_{1}=3.88 \mathrm{~J}$. 
The observed linearity in Fig. 5 where we have plotted $\ln \left[\left(\chi(T) T(\rho)-[\chi(T) T]_{0}(\rho)\right)(T / J)^{1 / 4}\right]$ vs. $(T / J)^{-1 / 2}$ for various $\rho$ confirms the validity of this low-temperature expansion. The free parameter $\alpha_{1}$ can be determined by fitting $\gamma(\rho)$ of Eq. 18 to the exact numerical Bethe ansatz solution of $[\chi(T) T](\rho)$ (Eq. 15). As shown in Fig. 6, for sufficiently small concentrations the fit turns out to be very good, giving $\alpha_{1}=3.88 \mathrm{~J}$.

A similar procedure can be used to determine the scaling behavior of the specific heat of segmented Heisenberg spin- $1 / 2$ chains. For the quenched case, one finds

$$
C \propto\left(\frac{T}{J}\right)^{-\frac{5}{4}} e^{-\gamma^{\prime} / \sqrt{T / J}}
$$

at sufficiently low temperatures. The corresponding effective gap $\gamma^{\prime}$ turns out to be

$$
\gamma^{\prime}=\gamma_{0}+\sqrt{4 \frac{\alpha_{1}^{\prime}}{J} \ln \left(\frac{1}{1-\rho}\right)},
$$

where a fit of the numerical data yields $\gamma_{0}=0.182$ and $\alpha_{1}^{\prime}=4.106 \mathrm{~J}$.

\section{CONCLUSIONS}

In summary, we have examined the spectral and thermodynamic response of various ensembles of segmented antiferromagnetic Heisenberg spin-1/2 chains. We have calculated the dynamical spin structure factor which can be probed by Neutron scattering experiments. The particular dependence of this quantity on the impurity concentration is determined by the distribution of segment lengths. However, there are several generic features which are observed for the most common distribution functions. These are (i) a rapid decay of the integrated spectral weight with increasing impurity concentration, (ii) a suppression of low-frequency poles (pseudo-gap), and (iii) the emergence of a discrete pole structure at higher energies, dominated by the smallest contributing clusters in the average. While we find that the first two predictions are consistent with the presently available experimental data, point (iii) might be the hardest to verify because the corresponding signals in a highly disordered sample are typically rather small and broad.

Two main contributions to the low-temperature thermodynamic response of segmented chains can be identified: a dominant divergent component from the odd-side clusters and a sub-dominant exponentially activated component due to the even-site clusters. The sub-dominant contribution is analyzed by subtracting the impurity tail from the total response function, as it is commonly done in the analysis of experiments. 1928 Assuming a certain type of distribution function, the corresponding effective gaps, here $\gamma$ for the uniform susceptibility and $\gamma^{\prime}$ for the specific heat, can be calculated. An analysis of these gaps can thus be used as an indicator of the underlying distribution function of segment lengths.

Complete segmentation of quantum spin chains, as it has been treated in this work, can be viewed as an extreme case of impurity scattering with an infinitely large on-site repulsive potential. While this mechanism may indeed lead to segmentation, in many physical realizations longer-range exchange paths exist which can partially restore extended states of the undoped parent systems. Consider for example $\mathrm{Zn}$-doped $\mathrm{CuGeO}_{3}$. This compound is known to have sizeable next-nearest neighbor exchange interactions along the $\mathrm{CuO}_{2}$-chain direction, giving rise to an effective $J_{1}-J_{2}$ model. Below the transition temperature $T_{S P}$ the compound goes into a dimerized spin-Peierls phase, whereas above $T_{S P}$ it is in a critical quasi-one-dimensional state. This spin-Peierls transition is suppressed upon replacing the $\mathrm{Cu}$-atoms randomly with $\mathrm{Zn} 29$, most likely because the partial segmentation due to the non-magnetic impurities impedes the quantum-critical extended states within the chains which in turn are a prerequisite for Peierls transitions. Because of the longer-range exchange paths $J_{2}$, these extended states are not completely destroyed, and a remnant spin-Peierls phase is observed in $\mathrm{Cu}_{1-\mathrm{x}} \mathrm{Zn}_{\mathrm{x}} \mathrm{GeO}_{3}$ at sufficiently small impurity concentrations $\mathrm{x} .2 \mathrm{~g}$

The segmentation of critical one-dimensional system competes with transitions such as three-dimensional ordering due to small inter-chain interactions or Peierls-type transitions. Segmented one-dimensional phases are more stable against low-temperature ordering transitions, and critical states can in turn be created in a controlled manner by impurity-doping short-range ordered systems, for example by intrefucing non-magnetic sites into quasi-onedimensional spin liquids such as two-leg spin-1/2 Heisenberg ladders.16.13.30

We thank A. Honecker and B. Normand for useful discussions, and acknowledge the Zumberge Foundation for financial support. 
${ }^{1}$ E. Abrahams, P. W. Anderson, D. C. Licciardello, and T. V. Ramakrishnan, Phys. Rev. Lett. 42, 673 (1979).

${ }^{2}$ A notable exception are the quasi-1D Quantum Hall edge currents which can circumvent surface impurities.

${ }^{3}$ S. Eggert, I. Affleck, and M. Takahashi, Phys. Rev. Lett. 73, 332 (1994).

${ }^{4}$ M. Troyer, H. Tsunetsugu, and D. Wuertz, Phys. Rev. B 50, 13515 (1994).

${ }^{5}$ E. Dagotto and T. M. Rice, Science 271, 618 (1996).

${ }^{6}$ T. M. Rice. S. Haas, M. Sigrist, and F. C. Zhang, Phys. Rev. B 56, 14655 (1997).

${ }^{7}$ K. Damle and S. Sachdev, Phys. Rev. B 57, 8307 (1998).

${ }^{8}$ S. Wessel and S. Haas, cond-mat/9905331 and cond-mat/9910259.

9 T. Giamarchi and A. M. Tsvelik, Phys. Rev. B 59, 11398 (1999).

${ }^{10}$ C. A. Doty and D. S. Fisher, Phys. Rev. B 45, 2167 (1992).

${ }^{11}$ S. Haas, J. Riera, and E. Dagotto, Phys. Rev. B 48, 13174 (1993).

${ }^{12}$ H. Röder, J. Stolze, R. N. Silver, and G. Müller, J. Appl. Phys. 79, 4632 (1996).

${ }^{13}$ E. Westerberg, A. Furusaki, M. Sigrist, and P. A. Lee, Phys. Rev. Lett. 75, 4302 (1995).

${ }^{14}$ P. W. Brouwer, C. Mudry, B. D. Simons, and A. Altland, Phys. Rev. Lett. 81, 862 (1998).

15 This response to disorder scattering is in contrast to random magnetic fields which generally cause exponential decay in the correlation functions.

${ }^{16}$ M. Sigrist and A. Furusaki, J. Phys. Soc. Jpn. 65, 2385 (1996).

17 G. B. Martins, M. Laukamp, J. Riera, and E. Dagotto, Phys. Rev. Lett. 78, 3563 (1997); M. Laukamp, G. B. Martins, C. Gazza, A. V. Malvezzi, E. Dagotto, P. M. Hansen, A. C. Lopez, and J. Riera, Phys. Rev. B 57, 10755 (1998).

${ }^{18}$ S. Haas, Phys. Rev. Lett. 80, 4052 (1998).

${ }^{19}$ H. Yamazaki and K. Katsumata, Phys. Rev. B 54, R6831 (1996); H. Asakawa et al., Phys. Rev. B 57, 8285 (1998).

${ }^{20}$ Z. Hiroi, S. Amelinckx, G. Van Tendeloo, and N. Kobayashi, Phys. Rev. B 54, 15849 (1996).

${ }^{21}$ Consider for example the energy of a 2 -site and a 4 -site antiferromagnetic spin- $1 / 2$ Heisenberg chain $(\mathrm{E}=-0.75 \mathrm{~J}-1.616 \mathrm{~J}=$ $-2.366 \mathrm{~J})$ vs. the energy of two 3 -site clusters $(\mathrm{E}=-\mathrm{J}-\mathrm{J}=-2 \mathrm{~J})$.

${ }^{22}$ C. Lanczos, J. Res. Natl. Bur. Stand. 45, 255 (1950).

${ }^{23}$ I. Affleck, D. Gepner, H. J. Schulz, and T. Ziman, J. Phys. A: Math. Gen. 22, 511 (1989).

${ }^{24}$ F. C. Alcaraz, M. N. Barber, and M. T. Batchelor, Phys. Rev. Lett. 58, 771 (1987); F. C. Alcaraz and M. J. Martins, Phys. Rev. Lett. 61, 1529 (1988).

${ }^{25}$ K. Nomura, Phys. Rev. B 48, 16814 (1993).

${ }^{26}$ F. Woynarovich and H. P. Eckle, J. Phys. A 20, L97 (1987); M. Karbach and K. H. Mütter, J. Phys. A 28, 4469 (1995). These references also discuss the significance of higher-order logarithmic corrections to Eq. (1) which have been neglected in this work, because a comparable accuracy for the pole weights is not accessible from fits to numerical diagonalization results.

${ }^{27}$ D. Stauffer and A. Aharony, "Introduction to Percolation Theory", (Taylor \& Francis, 1992).

${ }^{28}$ M. Azuma et al., Phys. Rev. Lett. 73, 3463 (1994).

29 M.C. Martin et al., Phys. Rev. B 56, 3173 (1997).

${ }^{30}$ B. Frischmuth and M. Sigrist, Phys. Rev. Lett. 79, 147 (1998). 